\title{
BMJ Open Antimicrobial use across six referral hospitals in Tanzania: a point prevalence survey
}

\author{
Jeremiah Seni (D) , Siana G Mapunjo, ${ }^{2}$ Rachel Wittenauer, ${ }^{3}$ Richard Valimba, ${ }^{4}$ \\ Andy Stergachis, ${ }^{3,5}$ Brian J Werth, ${ }^{5}$ Samir Saitoti, ${ }^{6}$ Noel H Mhadu, ${ }^{2}$ Edgar Lusaya, ${ }^{4}$ \\ Niranjan Konduri (iD) ${ }^{7}$
}

To cite: Seni J, Mapunjo SG,

Wittenauer $\mathrm{R}$, et al.

Antimicrobial use across six referral hospitals in Tanzania: a point prevalence survey. BMJ Open 2020;10:e042819. doi:10.1136/ bmjopen-2020-042819

- Prepublication history and additional material for this paper is available online. To view these files, please visit the journal online (http://dx.doi.org/10. 1136/bmjopen-2020-042819).

Received 21 July 2020 Revised 15 0ctober 2020 Accepted 13 November 2020

Check for updates

(c) Author(s) (or their employer(s)) 2020. Re-use permitted under CC BY-NC. No commercial re-use. See rights and permissions. Published by BMJ.

For numbered affiliations see end of article.

Correspondence to Dr Jeremiah Seni; senijj80@gmail.com

\section{ABSTRACT}

Objective To delineate the prevalence and factors associated with antimicrobial use across six referral hospitals in Tanzania using WHO point prevalence survey (PPS) methodology to inform hospital-specific antimicrobial stewardship programmes.

Design Cross-sectional analytical study.

Setting Six referral hospitals in Tanzania.

Participants Patients irrespective of age and gender $(n=948)$ admitted in the six referral hospital wards before 8:00 hours on each day of the survey were included in December 2019. Using the WHO PPS methodology, data on hospitals, wards, patients, antibiotics, and indications for antibiotics were collected.

Outcome measures We analysed the prevalence of antibiotic use by referral hospital, ward, indication and patient characteristics as the main outcomes. We also described adherence to the Tanzania Standard Treatment Guidelines (STG) and WHO's AWaRe categorisation of antibiotics.

Results Approximately $62.3 \%$ of inpatients were prescribed antibiotics, predominantly from the Access group of antibiotics (ceftriaxone, metronidazole or ampicillin-cloxacillin). The overall adherence of antibiotic prescriptions to the Tanzania STG was high $(84.0 \%)$, with the exception of Sekou Toure Regional Referral Hospital (68.0\%) and Maweni Regional Referral Hospital (57.8\%). The most common indication for antibiotic prescriptions was community-acquired infections $(39.8 \%)$. Children less than 2 years of age (OR $1.73,95 \% \mathrm{Cl} 1.02$ to $2.92, \mathrm{p}=0.039$ ); admission to surgical wards (OR $4.90,95 \% \mathrm{Cl} 2.87$ to $8.36, \mathrm{p}$ $<0.001$ ); and admission to paediatric wards (OR 3.93 , $95 \% \mathrm{Cl} 2.16$ to $7.15, \mathrm{p}<0.001$ ) were associated with increased odds of antibiotic use. Only 2 of 591 patients were prescribed antibiotics based on culture and antimicrobial susceptibility testing results.

Conclusions Empirical use of antibiotics is common, and the Access group of antibiotics is predominantly prescribed in children less than 2 years and patients admitted to surgical and paediatric wards. Lack of utilisation of antimicrobial susceptibility testing services in these hospitals requires urgent interventions. Routine monitoring of antibiotic use is recommended to be part of antibiotic stewardship programmes in Tanzania.

\section{Strengths and limitations of this study}

- This Point Prevalence Survey has addressed one of the five strategic objectives of the Tanzania National Action Plan on Antimicrobial resistance (2017-2022) and has created benchmarking information to guide antimicrobial stewardship programmes.

- Data were collected from six referral hospitals in Tanzania connoting regional-wide representation of antibiotic use with a large sample size $(n=948)$.

- This survey was based on the standardised WHO methodology and involvement of staff in these referral hospitals will enable them to conduct similar surveys to monitor temporal antibiotic use.

- We could not depict seasonal variations in antibiotics use as this was a point prevalence survey.

- It was not possible to delineate the contribution of prescribers' professional ranks to antibiotic prescription because some patients were prescribed multiple antibiotics by different prescribers.

\section{INTRODUCTION}

Antimicrobial resistance (AMR) is an escalating threat to global health, endangering the ability to prevent and cure a wide range of infectious diseases. ${ }^{1}$ The current AMR global crisis is the result of a number of factors, with inappropriate use of antibiotics and lack of antimicrobial use surveillance systems being core factors contributing to the spread of AMR. ${ }^{23}$ The 2016 Global Health Security Agenda assessment concluded that AMR is a major problem in Tanzania and that there are high levels of inappropriate use of antimicrobials in the human and animal sectors. ${ }^{4}$ Inappropriate antimicrobial use is likely accelerating the spread of AMR, which has clinical and cost implications for patients, communities and the healthcare system. ${ }^{56}$ The development of national monitoring systems is an essential part of national action plans for AMR, as clearly stipulated in the Global Action Plan and the Tanzanian National Action Plan on AMR. ${ }^{7-9}$ Hospitals 
are excellent settings for gaining understanding of antibiotic prescribing patterns. ${ }^{10-12}$ Collecting hospital data on antimicrobial use and subsequently implementing informed interventions to optimise antibiotic use has significant potential to lower antibiotic resistance rates at local levels.

The WHO Methodology for Point Prevalence Survey (PPS) on antibiotic use in hospitals ${ }^{13}$ is a standardised tool that allows for comparison of results within and among countries. ${ }^{14-16}$ A large study involving 53 countries reported a lower proportion of antibiotic use in North American and European countries $(<40.0 \%)$, whereas the overall proportion of antibiotic use in African countries was $50.0 \% .{ }^{17}$ Because there is limited information on antibiotic use that used WHO's PPS methodology in the East African Region and Tanzania in particular, ${ }^{18}$ the primary objective of the present study was to collect information on antibiotic prescriptions in selected referral hospitals to estimate the prevalence of antibiotic use in these hospitals. A secondary objective was to use this information to inform interventions at the local and national levels aimed at improving antibiotic prescribing, antibiotic use and antimicrobial stewardship (AMS) programmes.

\section{METHODS}

\section{Study design, settings and duration}

This PPS on antibiotic use was conducted in December 2019 at six referral hospitals in the United Republic of Tanzania. Mbeya Zonal Referral Hospital (ZRH) is located in the Southern Highlands with 553 total bed capacity and 323536 total hospital annual admissions, and 346 hospital beds were included in the survey. The respective values for the other five referral hospitals were Benjamin Mkapa ZRH (Central area): 400, 5934 and 67; Sekou Toure Regional Referral Hospital (RRH) (Lake Victoria area): 315, 22068 and 226; Temeke RRH (Costal area): 309, 11 792 and 224; Bukoba RRH (Northern area): 308, 11009 and 163; and Maweni RRH (Western area): 168, 9121 and 54. These hospitals provide services to patients referred from lower tiers of healthcare facilities (ie, district hospitals, health centres and dispensaries). However, a ZRH serves as a tertiary hospital with more specialty services and also provide services to patients referred from RRHs.

\section{Study population, inclusion and exclusion criteria}

In accordance with the WHO methodology for PPS, all patients (irrespective of age and gender) admitted in the referral hospital wards before 8:00 hours on the day of the survey were included. ${ }^{13}$ Only antibiotics administered through oral, parenteral, rectal or inhalation routes were included. The WHO PPS methodology recommends that for hospitals with $<500$ total inpatient beds, all patients meeting the inclusion criteria must be surveyed. Since hospital bed size in all six surveyed hospitals did not exceed 500 beds, no sample size estimation was needed, and all patients on the day of survey were enrolled. ${ }^{13}$
Inpatients with incomplete data in their medical files were excluded from the PPS.

\section{Sampling methods and sample size}

The six prioritised referral hospitals were chosen based on multiple criteria for ongoing, cross-cutting interventions on infection prevention and control (IPC) measures and AMS. These included: (1) country's zonal/regional representation; (2) presence of hospital staff recently trained on the new IPC guidelines; (3) presence of any other implementing partner performing AMR surveillancerelated work and (4) hospitals that were not involved in a previously conducted PPS by WHO and the Ministry of Health, Community Development, Gender, Elderly and Children in Tanzania. These six hospitals were approved as intervention sites by the National AMR Multi-sectoral Coordination Committee. Data collection was completed in each hospital within a week after the first day of data collection. To minimise the impact of patients moving between wards, each ward was completely surveyed in 1 day. Of the 2053 total beds in the six referral hospitals surveyed, 1080 patients were eligible (the remaining beds were unoccupied). Of these, a total $948(87.8 \%)$ were included for data analysis. The remaining patients were not enrolled because of incomplete and/or mismatching of information in the medical files/records.

\section{Data collection}

A comprehensive training on the protocol for PPS for participating hospital staff involving doctors, pharmacists and nurses and staff from the Ministry of Health, Community Development, Gender, Elderly and Children (deemed research assistants in this paper) was conducted by the coinvestigators in Dar es Salaam, Tanzania, 10 December 2019-13 December 2019, prior to data collection 16 December 2019-21 December 2019. One component of the training was a session on the protection of study participants, with an emphasis on data confidentiality, patient privacy, and hospital staff privacy. The training sessions included practising with and pilot testing the data collection forms and methods.

Antimicrobial drug regimens prescribed to patients hospitalised at or before 08:00 hours on the days of the PPS were abstracted from patient files and other medical records. The records of all patients meeting the eligibility criteria were included in the PPS irrespective of whether they received antibiotic treatment. Antibiotics were classified according to the Anatomical Therapeutic Chemical Classification (ATC) methodology developed by the WHO Collaborating Centre for Drug Statistics Methodology in Oslo, Norway, ${ }^{19}$ and by the 2019 WHO AWaRe (Access, Watch and Reserve) classification of antibiotics. ${ }^{20}$

Study endpoints were the prevalence of antibiotic use, WHO's AWaRe categorisation of antibiotics, ward, indication and patient characteristics. No patient interviews and no clinician or health facility staff interviews were performed. Any engagement with health facility staff was only for the purpose of retrieval of medical records. 


\section{Data management}

Data were entered in a standardised MS Excel template. The data were subjected to a quality check process and were subsequently analysed using STATA (software V.13.0) in accordance with the objectives of the PPS. Each spreadsheet was uploaded to a secure, password-protected Microsoft One-Drive that could only be accessed by the investigators. Only deidentified patient and provider data were recorded. Core variables collected were specified in the WHO PPS methodology for each of the following categories: hospital variables, ward variables, patient variables (anonymised), prescribers' professional ranks, indication of antibiotic treatment variables and prescribed antibiotics variables. Antimicrobial use was defined as types and quantities of antimicrobials used by a patient in a hospital during a specific period of time. Continuous variables were described as mean $\pm \mathrm{SD}$ or median (plus IQR), depending on the distribution of data. Categorical variables were described and presented in frequencies and percentages (proportions). These data allowed disaggregation of outcome variable (ie, antibiotic use) by patient characteristics (ie, gender and age), wards, hospitals and antibiotic treatment indication. Univariate and multivariate logistic regression analyses were done to ascertain the independent predictor variables for antibiotic use using OR, 95\% CI. Any factor with a $\mathrm{p} \leq 0.05$ in univariate analysis was subjected to multivariate analysis. A p $\leq 0.05$ was considered to be statistically significant.

Permission to conduct this PPS was provided by the respective regional and hospital authorities. As this PPS did not directly involve patients but rather their information in medical files/records, a waiver of consent was requested from the authorities of the participating hospitals. Trained research assistants from the national level and local referral hospitals' health personnel were involved to help build a foundation for continuous monitoring of antibiotic prescription and use in the context of strengthening AMS programmes.

\section{Patients and public involvement statement}

This PPS was conducted as part and parcel of the implementation of the Tanzania National Action Plan on AMR (2017-2022).

\section{RESULTS}

\section{Baseline demographic and clinical information of patients enrolled}

Data from 948 patients were included in this study, with female patients accounting for $58.3 \%$. The overall median age (IQR) among patients $\geq 2$ years of age was 32 $(22-47.5)$ years, whereas the median age for patients $<2$ years was 3 (1-10) months. Most of the patients were enrolled from Mbeya ZRH (36.2\%), and the majority had indwelling peripheral vascular catheters $(73.5 \%)$
Table 1 Demographic and clinical characteristics of enrolled patients at Tanzanian zonal hospitals, $n=948$

\begin{tabular}{|c|c|}
\hline Variable & No (\%) \\
\hline \multicolumn{2}{|l|}{ Gender } \\
\hline Male & $395(41.7)$ \\
\hline Female & $553(58.3)$ \\
\hline \multicolumn{2}{|l|}{ Hospital } \\
\hline Mbeya ZRH & $343(36.2)$ \\
\hline Temeke RRH & $176(18.6)$ \\
\hline Sekou Toure RRH & $171(18.0)$ \\
\hline Bukoba RRH & $140(14.8)$ \\
\hline Benjamin Mkapa ZRH & $64(6.8)$ \\
\hline Maweni RRH & $54(5.7)$ \\
\hline \multicolumn{2}{|l|}{ Wards } \\
\hline Adult medical & $267(28.2)$ \\
\hline Adult surgical & $119(12.6)$ \\
\hline Paediatrics & $140(14.8)$ \\
\hline Intensive care unit & $45(4.8)$ \\
\hline Mixed & $377(39.8)$ \\
\hline \multicolumn{2}{|l|}{ McCabe score } \\
\hline Non-fatal & $817(86.2)$ \\
\hline Rapidly fatal & $24(2.5)$ \\
\hline Ultimately fatal & $73(7.7)$ \\
\hline Not reported & $34(3.6)$ \\
\hline Presence of central vascular catheter & $14(1.5)$ \\
\hline Presence of peripheral vascular catheter & $697(73.5)$ \\
\hline Indwelling urinary catheter & $282(29.7)$ \\
\hline Patients intubated & $20(2.1)$ \\
\hline $\begin{array}{l}\text { Patients with malaria during current } \\
\text { hospitalisation }\end{array}$ & $79(8.3)$ \\
\hline $\begin{array}{l}\text { Patients with tuberculosis during current } \\
\text { hospitalisation }\end{array}$ & 22 (2.3) \\
\hline Patients with HIV infections & $61(6.4)$ \\
\hline $\begin{array}{l}\text { Previous history of hospitalisation (within } \\
90 \text { days) }\end{array}$ & $84(8.9)$ \\
\hline
\end{tabular}

$\mathrm{RRH}$, regional referral hospital; $\mathrm{ZRH}$, zonal referral hospital.

(table 1). Approximately one-quarter of patients were referred from other hospitals $(26.4 \%, \mathrm{n}=250)$.

\section{Antibiotic use}

A total of 591 (62.3\%) patients were receiving antibiotic treatment during the PPS. Hospital-specific proportions of patients receiving antibiotics ranged from $51.3 \%$ in Mbeya ZRH to $74.3 \%$ in Sekou Toure RRH (figure 1). The majority of patients on antibiotics were given one $(37.1 \%, \mathrm{n}=219)$ or two agents $(54.8 \%, \mathrm{n}=324)$. Three, four and five antibiotics were prescribed in $6.9 \% \quad(n=41)$, $0.9 \%(n=5)$ and $0.3 \%(n=2)$ of cases, respectively. Only two patients were specifically treated based on antimicrobial susceptibility test (AST) laboratory results. 


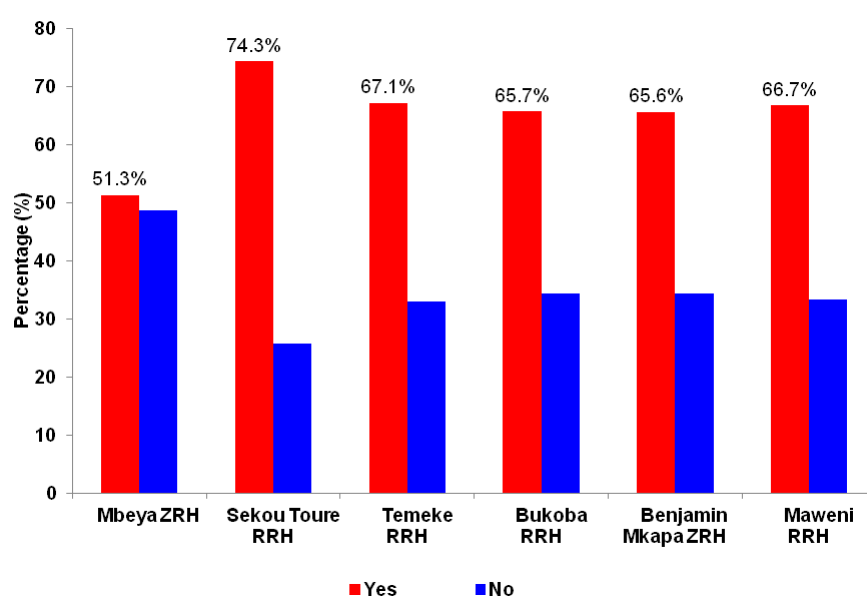

Figure 1 Proportions of antibiotic use across six referral hospitals. RRH, regional referral hospital; ZRH, zonal referral hospital.

\section{Distribution of antibiotic prescriptions by referral hospital and ward}

A total of 1013 antibiotics were prescribed to patients during the PPS. The most commonly used antibiotics were ceftriaxone $(30.9 \%)$, metronidazole $(22.9 \%)$, ampicillin-cloxacillin $(17.0 \%)$ and gentamicin $(11.0 \%)$. Ceftriaxone, metronidazole and gentamicin were prescribed in all hospitals, whereas vancomycin, moxifloxacin and meropenem were prescribed only at ZRHs. Antibiotic prescriptions were predominantly in the mixed ward $(\mathrm{n}=389,38.4 \%)$, followed by adult medical wards $(\mathrm{n}=205$, $20.2 \%)$ and paediatric wards $(\mathrm{n}=194,19.2 \%)$ (table 2).

\section{Diagnoses underlying the indication for antibiotic therapies}

Most antibiotics were used for community-associated infections (36.7\%), followed by surgical prophylaxis $(30.2 \%)$ and medical prophylaxis $(24.0 \%)$. Ceftriaxone and metronidazole were predominant antibiotics regardless of the indications (table 3 ).

A total of 670 diagnoses underlying the indication for antibiotic therapies were recorded. The most common diagnoses was skin, soft tissue, bone and joint infections $(17.2 \%, \mathrm{n}=115)$ and gastrointestinal tract infections $(12.7 \%, \mathrm{n}=85)$. Other diagnoses were obstetric and gynaecological infections $(10.9 \%, \mathrm{n}=73)$; cardiovascular system infections $(10.7 \%, \mathrm{n}=72)$; respiratory tract infections $(10.4 \%, \mathrm{n}=70)$; central nervous system infections $(4.6 \%, \mathrm{n}=31)$; urinary tract infections $(3.0 \%, \mathrm{n}=20)$ and other infections $(6.7 \%, \mathrm{n}=45)$. However, antibiotic use for a purpose other than treatment was reported in $127(19.0 \%)$ patients and in 32 diagnoses $(4.8 \%)$ where there was a completely undefined site with no systemic inflammation.

\section{Adherence to the Tanzania standard treatment guidelines}

Of the 1013 antibiotics assessed, information on adherence was reported in 998 antibiotics, and the overall compliance was $84.0 \%$. The highest compliance rates were observed in Temeke RRH (99.5\%), Mbeya ZRH (94.0\%) and Bukoba RRH (90.2\%). Adherence rates at the remaining hospitals were Benjamin Mkapa ZRH $(72.4 \%)$, Sekou Toure RRH $(68.0 \%)$ and Maweni RRH $(57.8 \%)$.

\section{Aware classification of antibiotics}

A total of $991(97.9 \%), 18(1.8 \%)$ and $3(0.3 \%)$ antibiotics were classified as Access, Watch and Reserve, respectively. Interestingly, all three antibiotics in the Reserve group were prescribed in the ZRH (see online supplemental table 1).

\section{Factors associated with antibiotic use}

Factors associated with increased odds of antibiotic use were age less than 2 years, male gender and type of hospital ward. Admission to Mbeya ZRH and patients with no previous admission within 90 days were associated with decreased odds of antibiotic use. The multivariate logistic regression analysis showed that independent predictors for antibiotic use were children less than 2 years of age (OR 1.73, 95\% CI 1.02 to 2.92, $\mathrm{p}=0.039$ ); admission to surgical wards (OR 4.90, 95\% CI 2.87 to $8.36, \mathrm{p}<0.001$ ); and admission to paediatric wards (OR 3.93, 95\% CI 2.16 to $7.15, \mathrm{p}<0.001$ ) (table 4 ).

\section{DISCUSSION}

Monitoring of antibiotic use in hospitals is a pivotal component of AMS programmes and is crucial to informing national and hospital policies and practices aimed at improving antibiotic use and guiding procurement of these agents. In this PPS, empirical use of antibiotics was high $(62.3 \%)$, with subtle variations across hospitals. Similarly, high proportions of antibiotic use have been previously reported in Kenya (67.7\%), Botswana (70.6\%), Nigeria $(69.7 \%)$ and Jordan $(78.2 \%) \cdot{ }^{18-23}$ In contrast to these findings, low proportions of antibiotics use have been reported in South Africa (37.7\%), Brazil (42.5\%), UK $(40.9 \%)$, Northern Ireland $(46.2 \%)$ and Belgium $(27.1 \%) .{ }^{14} 23-26$ The differences can be accounted for by varying stringency pertaining to antimicrobial use guidelines/policies across countries, differences in patients' characteristics, and the need to administer antibiotics in patients referred to tertiary and specialised hospitals. In Tanzania, previous studies (each based on one centre) that used a non-PPS methodology also reported a high proportion of antibiotic use $(42.6 \%-84.9 \%) .{ }^{5112728}$

The most common antimicrobial agents reported in this PPS were ceftriaxone and metronidazole, regardless of the hospitals or wards/units where the patients were admitted and the indications for antibiotic prescriptions. These agents are relatively inexpensive and widely available in Tanzania and are used routinely for surgical prophylaxis and treatment of invasive infections. They are also Access antibiotics listed in the Standard Treatment Guidelines. ${ }^{29}$ This is consistent with findings from two other studies in Tanzania. ${ }^{27} 28$ On the other hand, vancomycin (Watch antibiotic) and moxifloxacin and meropenem (Reserve antibiotics in the Standard Treatment Guidelines) were 


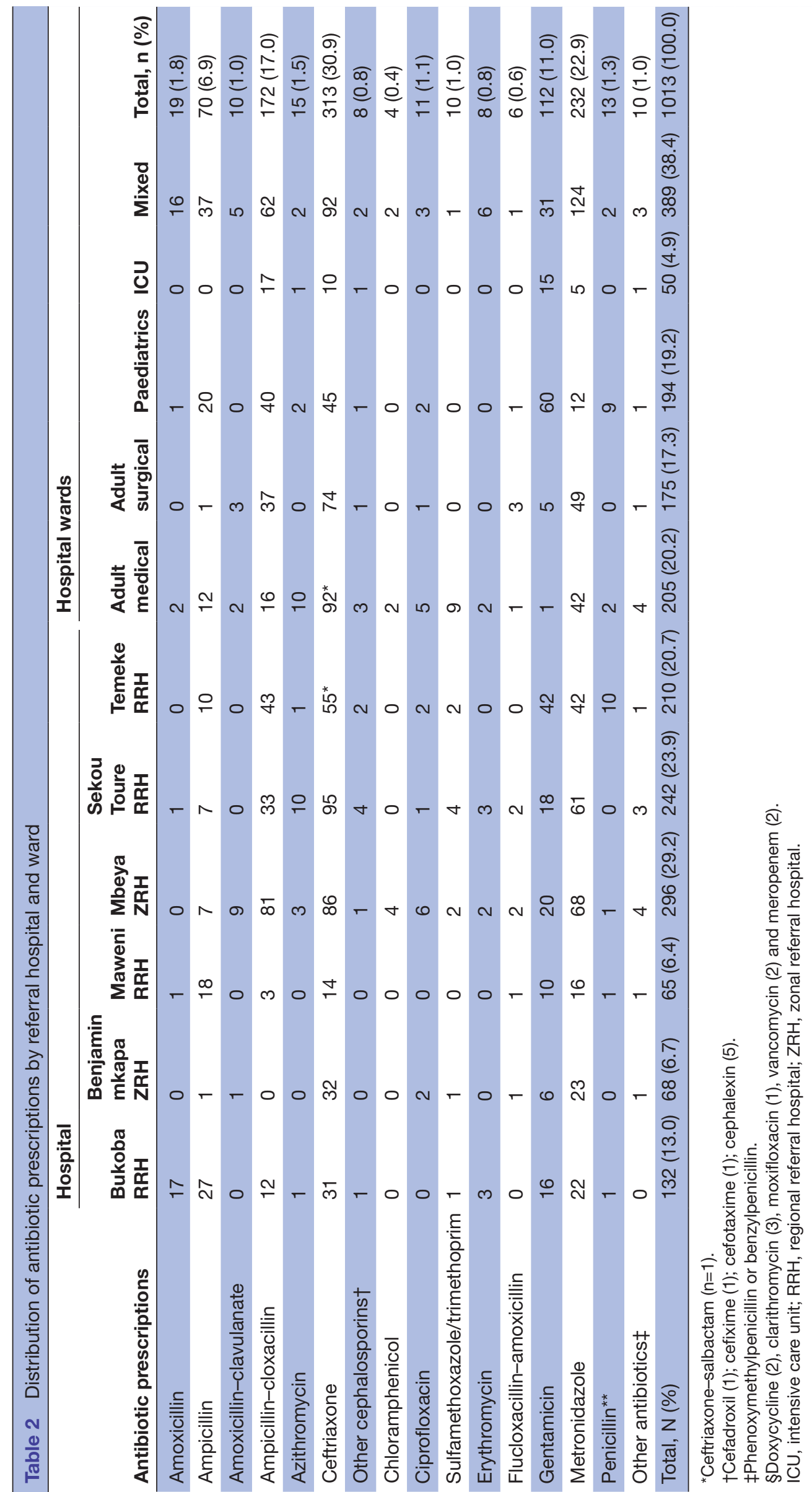


Table 3 Distribution of antibiotic prescriptions by indications

\begin{tabular}{|c|c|c|c|c|c|c|}
\hline \multirow[b]{2}{*}{ Antibiotic prescriptions } & \multicolumn{6}{|c|}{ Indications } \\
\hline & CAI & HAl & $\begin{array}{l}\text { Medical } \\
\text { prophylaxis }\end{array}$ & $\begin{array}{l}\text { Surgical } \\
\text { prophylaxis }\end{array}$ & $\begin{array}{l}\text { Other } \\
\text { indications }\end{array}$ & Total, n (\%) \\
\hline Amoxicillin & 6 & 1 & 6 & 4 & 2 & $19(1.8)$ \\
\hline Ampicillin & 29 & 5 & 13 & 17 & 4 & $68(6.6)$ \\
\hline Amoxicillin-clavulanate & 4 & 0 & 0 & 6 & 0 & $10(1.0)$ \\
\hline Ampicillin-cloxacillin & 53 & 7 & 60 & 58 & 3 & $181(17.5)^{\star}$ \\
\hline Azithromycin & 10 & 0 & 4 & 0 & 0 & $14(1.4)$ \\
\hline Ceftriaxone & $141 \S$ & 18 & 57 & 95 & 7 & $318(30.8)^{\star}$ \\
\hline Other cephalosporins & 6 & 0 & 1 & 0 & 1 & $8(0.8) \dagger$ \\
\hline Chloramphenicol & 1 & 1 & 1 & 1 & 0 & $4(0.4)$ \\
\hline Ciprofloxacin & 6 & 1 & 3 & 2 & 0 & $12(1.2)^{*}$ \\
\hline Sulfamethoxazole/trimethoprim & 9 & 0 & 1 & 0 & 0 & $10(1.0)$ \\
\hline Erythromycin & 4 & 1 & 2 & 1 & 0 & $8(0.8)$ \\
\hline Flucloxacillin-amoxicillin & 2 & 0 & 2 & 1 & 1 & $6(0.6)$ \\
\hline Gentamicin & 49 & 8 & 48 & 9 & 0 & $114(11.0)^{\star}$ \\
\hline Metronidazole & 76 & 13 & 34 & 102 & 12 & $237(22.9)^{\star}$ \\
\hline Penicillin $\ddagger$ & 11 & 0 & 0 & 0 & 2 & $13(1.2)$ \\
\hline Other antibiotics§ & 4 & 1 & 3 & 1 & 1 & $10(1.0)^{*}$ \\
\hline Total, N (\%) & $411(39.8)$ & $56(5.4)$ & $235(22.8)$ & $297(28.8)$ & $33(3.2)$ & 1032 (100.0)ף \\
\hline
\end{tabular}

*Antibiotics used for multiple indications.

†Cefadroxil (1); cefixime (1); cefotaxime (1); cephalexin (5).

$\ddagger$ Phenoxymethylpenicillin or benzylpenicillin.

$\S$ Ceftriaxone-salbactam $(n=1)$.

ПClarithromycin (3), doxycycline (2), meropenem (2), vancomycin (3).

$\mathrm{CAl}$, community-associated infection; HAI, hospital-associated infection.

used in Benjamin Mkapa ZRH and Mbeya ZRH in five of 1013 prescriptions. The predominance of ceftriaxone and metronidazole and the small number of vancomycin and meropenem prescriptions are comparable to other studies from Nigeria, Botswana and Kenya, indicating similar economical, epidemiological and clinical predispositions in these sub-Saharan African countries. ${ }^{18} 2122$ Restricted use of meropenem and vancomycin is appropriate because these are the last-line treatment options for severe invasive infections caused by gram negative and gram positive bacteria, respectively. The most common indications for antibiotic prescriptions were communityacquired infections, surgical prophylaxis and medical prophylaxis. These indications are also tallying with the predominant diagnoses of skin, soft tissue, bone and joint infections and gastrointestinal tract infections.

High adherence of prescriptions to the standard treatment guidelines in the current study $(84.0 \%)$ is similar to previous studies in South Africa $(98.0 \%)$, Northern Ireland $(72.0 \%-81.8 \%)$, Jordan $(92.2 \%-92.7 \%)$, Brazil $(76.5 \%-87.3 \%)$ and Belgium $(76.6 \%) .{ }^{23-26}$ In contrast to these findings, a study in Nigeria found low adherence, ranging from $0.3 \%$ to $7.2 \%{ }^{22}$ The finding of high adherence in Mbeya ZRH (94.0\%), in contrast to the $63.0 \%$ found in 2018 by another study in the same hospital, may be accounted for by the possibility of postsurvey sensitisation and/or awareness. ${ }^{28}$ This is further exemplified by the low proportion of antibiotic use in this hospital, which is in contrast to all other hospitals. The findings of moderate adherence to the Standard Treatment Guidelines in Sekou Toure RRH and Maweni RRH call for in-depth analysis on the underlying causes and provision of specific remedial measures.

Despite the availability of AST services in four of the six surveyed referral hospitals, only two patients were specifically treated based on laboratory results. There is generally poor availability of AST in hospitals in resourcelimited countries and limited availability of trained staff. In Tanzania, only 7 of 39 hospitals reported to conduct AST and keep surveillance reports. ${ }^{30}$ Therefore, in our study, the root causes of low AST services utilisation need to be delineated both at individual and/or hospital levels. For example; is it cost for AST services which hinder patients' access? Or constraints in laboratory supplies which limit laboratory technologists/scientists to perform AST after isolating a pathogen? Or is it lack of documentation of the laboratory results to the patients' files in the wards? Or just a coincidental finding based on the nature of the PPS methodology? In contrast to our findings, appreciable numbers of patients were managed based on laboratory 
Table 4 Factors associated with antibiotic use among patients in six hospitals

\begin{tabular}{|c|c|c|c|c|c|}
\hline \multirow[b]{2}{*}{ Variable (N) } & \multirow[b]{2}{*}{ Antibiotic use (n, (\%)) } & \multicolumn{2}{|l|}{ Univariate analysis } & \multicolumn{2}{|c|}{ Multivariate analysis } \\
\hline & & $95 \% \mathrm{Cl}$ & $P$ value & $95 \% \mathrm{Cl}$ & $P$ value \\
\hline \multicolumn{6}{|l|}{ Age category (years) } \\
\hline$<2(167)$ & $132(79.0)$ & 2.64 (1.78 to 3.94$)$ & $<0.001$ & 1.73 (1.02 to 2.92$)$ & 0.039 \\
\hline \multicolumn{6}{|l|}{ Gender } \\
\hline Female (553) & 328 (59.3) & $0.73(0.56$ to 0.96$)$ & 0.023 & $0.96(0.72$ to 1.29$)$ & 0.801 \\
\hline \multicolumn{6}{|l|}{ Hospital ${ }^{*}$} \\
\hline Bukoba RRH (140) & $92(65.7)$ & 1 & & & \\
\hline Benjamin Mkapa ZRH (64) & $42(65.6)$ & 0.99 (0.53 to 1.86$)$ & 0.990 & & \\
\hline Temeke RRH (176) & $118(67.1)$ & $1.06(0.66$ to 1.70$)$ & 0.803 & & \\
\hline \multicolumn{6}{|l|}{ Ward } \\
\hline Adult medical (267) & $128(47.9)$ & 1 & & & \\
\hline Adult surgical (119) & $98(82.4)$ & 5.07 (2.99 to 8.60$)$ & $<0.001$ & 4.90 (2.87 to 8.36$)$ & $<0.001$ \\
\hline Paediatrics (140) & $118(84.3)$ & 5.82 (3.48 to 9.74$)$ & $<0.001$ & 3.93 (2.16 to 7.15$)$ & $<0.001$ \\
\hline Intensive care unit (45) & $29(64.4)$ & 1.97 (1.02 to 3.79$)$ & 0.043 & 1.23 (0.57 to 2.64$)$ & 0.595 \\
\hline Mixed (377) & $218(57.8)$ & 1.49 (1.09 to 2.04$)$ & 0.013 & 1.37 (0.99 to 1.90$)$ & 0.06 \\
\hline \multicolumn{6}{|l|}{ Previous hospitalisation† } \\
\hline No (650) & $426(65.4)$ & 1 & & & \\
\hline Yes (35) & 31 (88.6) & $0.90(0.31$ to 2.61$)$ & 0.841 & & \\
\hline \multicolumn{6}{|l|}{ Medical prophylaxis } \\
\hline No (479) & $434(90.6)$ & 1 & & & \\
\hline Yes (164) & $142(86.6)$ & $0.67(0.39$ to 1.15$)$ & 0.148 & & \\
\hline \multicolumn{6}{|l|}{ Surgical prophylaxis } \\
\hline No (474) & $419(88.4)$ & 1 & & & \\
\hline Yes (169) & $157(92.9)$ & 1.71 (0.90 to 3.29) & 0.103 & & \\
\hline \multicolumn{6}{|l|}{ Other indications not specified } \\
\hline No (620) & $557(89.8)$ & 1 & & & \\
\hline Yes (23) & $19(82.6)$ & $0.54(0.18$ to 1.63$)$ & 0.272 & & \\
\hline
\end{tabular}

*Hospital variable was removed in the multivariate model because of collinearity with ward. †Within 90 days and 214 were removed as their hospitalisation status was unknown.

$\mathrm{CAl}$, community-associated infections; HAI, hospital-associated infections; $\mathrm{RRH}$, regional referral hospital; $\mathrm{ZRH}$, zonal referral hospital.

results in South Africa, Brazil and Belgium. ${ }^{24-26}$ Therefore, to avert the empirical use of antibiotics in these referral hospitals, it is recommended that similar PSS be routinely conducted to assess the trend. Also, addressing hospital-based and/or individual-based factors hindering routine provision of this service in the context of the Tanzanian National Action Plan on AMR implementation is urgently needed. ${ }^{8}$ Future research should aim to identify the root causes of poor availability and uptake of AST through in-depth interview with the key stakeholders 
(ie, patients, prescribers, dispensers, laboratory experts, managers and other AMS champions).

It is well known that prescribers, patients and healthcare facilities' infrastructures can contribute to inappropriate antibiotic use, and therefore, judicious mitigation is required to ensure appropriate use of these agents and prevent AMR. ${ }^{31}$ In this PPS, children less than 2 years of age, admission to surgical wards and admission to paediatric wards were associated with increased odds of being prescribed antibiotics. Patients admitted to surgical wards are more likely to be given antibiotics before and after surgical interventions. On the hand, children's conditions are unpredictable and in some cases progression of infectious disease to fatal outcomes is common, and therefore, some clinicians opt to prescribe antibiotics empirically. Nevertheless, a need to collect appropriate samples for culture and antimicrobial susceptibility services prior to institution of antibiotics is reiterated to guide specific treatments. Children less than 15 years of age had increased odds of being given antibiotics in a study conducted in Eritrea. ${ }^{32}$ A study in China showed that patient pressure, time pressure, financial incentives and institutional environment can also predict antibiotic prescriptions and subsequent use. ${ }^{31}$

This is the first study from Tanzania that utilised the WHO PPS methodology and is being reported in the public domain. Tanzania was among five countries in the WHO African region that participated in the 2018 pilot for the WHO PPS methodology prior to its final release by WHO. Therefore, the institutional experience of the Tanzania Ministry of Health, Community Development, Gender, Elderly and Children on implementation of the WHO PPS methodology facilitated rapid transfer of learning and uptake by staff in the six hospitals. WHO's International Health Regulations Benchmark 3.4 on optimising use of antimicrobials recommended that member states monitor antimicrobial use, among other AMS activities, in designated health facilities to reach capacity level $3{ }^{33}$ As WHO's AMS practical toolkit recommends, our future interventions will ensure that a suitable governance structure, such as an AMS committee, exists. ${ }^{34}$ Hospital medicines and therapeutics committee are also recommended to be utilised as a platform for AMS interventions by the Pharmaceutical Services Unit of the Ministry of Health, Community Development, Gender, Elderly and Children. We anticipate that the participating hospitals will use the study findings through these governance structures and identify feasible targets for stewardship. In addition, our future interventions will assess why uptake of antimicrobial susceptibility services is limited and identify barriers and workflow solutions.

\section{Limitations}

Some of the limitations in this PPS were related to its design. For example, we could not depict seasonal variations in antibiotics use. However, the study has given a baseline assessment on which other studies that use the PPS methodology can be compared. It was not possible to delineate the contribution of prescribers' professional ranks to antibiotic prescription because some patients were prescribed multiple antibiotics by different prescribers. As designed, our study did not conduct any qualitative interviews with hospital physicians.

\section{CONCLUSIONS AND IMPLICATIONS}

Approximately $62.3 \%$ of inpatients were prescribed antibiotics empirically, with the predominance of ceftriaxone and metronidazole irrespective of wards/units, hospitals or indications. The adherence of antibiotic prescriptions to the Tanzania Standard Treatment Guidelines was encouraging $(84.0 \%)$ with the exception of Sekou Toure RRH and Maweni RRH. The most common indication was community-acquired infections. Children less than 2 years of age and admission to surgical and paediatric wards had increased odds of being prescribed antibiotics. These groups should be prioritised in the mitigation strategies for antibiotic stewardship. In this PPS, all referral hospitals involved are publicly owned and therefore future studies should focus on delineating antibiotic use in both public and private hospitals. Lack of utilisation of AST services in these hospitals is worrisome and requires urgent interventions.

\section{Author affiliations}

${ }^{1}$ Weill Bugando School of Medicine, Catholic University of Health and Allied Sciences, Mwanza, Tanzania

${ }^{2}$ Ministry of Health, Community Development, Gender, Elderly and Children, Dodoma, Tanzania

${ }^{3}$ Department of Global Health, University of Washington, Seattle, Washington, USA ${ }^{4}$ USAID Medicines, Technologies, and Pharmaceutical Services (MTaPS) Program,

Management Sciences for Health (MSH), Dar es Salaam, Tanzania

${ }^{5}$ School of Pharmacy, University of Washington, Seattle, Washington, USA

${ }^{6}$ University of Dodoma Hospital, Dodoma, Tanzania

${ }^{7}$ USAID Medicines, Technologies, and Pharmaceutical Services (MTaPS),

Management Sciences for Health (MSH), Arlington, Virginia, USA

Twitter Niranjan Konduri @N_Konduri

Acknowledgements The authors thank the 36 dedicated research assistants for their technical roles and the hospitals' management teams for their support and guidance. The following colleagues are greatly appreciated for their role in problem solving and ensuring adherence to the PPS protocol: Dr Alex Makalla, Dr Fortunata Atugonzile, Emiliana Francis, Martha Kikwale, Dr Jackson Ilangali, Daniel Pyuza, Samson S Langeni and Dr Pantaleo M Joseph. We gratefully acknowledge the leadership and support of Professor Muhammad Bakari Kambi, former Chief Medical Officer of Tanzania and Mr Daudi Msasi, Director of Pharmaceutical Services of the Ministry of Health,Community Development, Gender, Elderly and Children, and clinicians from all referral hospitals where this PPS was conducted.

Contributors JS, SGM, RV, AS, BJW, EL and NK had substantial contribution to the conception and design of the work; SM, AS and BJW led the training of the research assistants; JS, SGM, RV, SS, NHM, EL and NK participated in the data collection; JS, RW, SS and NHM played a critical role in the data management and data analysis; JS wrote the initial draft of the manuscript; SGM, RW, RV, AS, BJW, EL and NK critically reviewed and edited the draft manuscript; SGM, RV, EL and NK were responsible for coordinating all research activities; all authors approved the final draft.

Funding This study was made possible by the generous support of the American people through the US Agency for International Development (USAID) contract no. 7200AA18C00074. BJW is supported in part by the National Institute of Allergy and Infectious Diseases (R21Al132994 and R01Al136979).

Disclaimer The contents are the responsibility of the authors and do not necessarily reflect the views of USAID or the US Government. 
Competing interests BJW has received grant support from commercial sources, including Shionogi Inc. All other authors declare to have no any competing interests.

Patient consent for publication Not required.

Ethics approval Research and ethical approval was provided by the United Republic of Tanzania's National Health Research and Ethics Review Committee (Nat-HREC) of the National Institute for Medical Research (Reg. No. NIMR/HQ/R.8a/ Vol.IX/3297).

Provenance and peer review Not commissioned; externally peer reviewed.

Data availability statement Data are available on reasonable request from the national AMR focal person in the Ministry of Health, Community Development, Gender, Elderly and Children. Additional data from this PPS will be published by the coauthors.

Supplemental material This content has been supplied by the author(s). It has not been vetted by BMJ Publishing Group Limited (BMJ) and may not have been peer-reviewed. Any opinions or recommendations discussed are solely those of the author(s) and are not endorsed by BMJ. BMJ disclaims all liability and responsibility arising from any reliance placed on the content. Where the content includes any translated material, BMJ does not warrant the accuracy and reliability of the translations (including but not limited to local regulations, clinical guidelines, terminology, drug names and drug dosages), and is not responsible for any error and/or omissions arising from translation and adaptation or otherwise.

Open access This is an open access article distributed in accordance with the Creative Commons Attribution Non Commercial (CC BY-NC 4.0) license, which permits others to distribute, remix, adapt, build upon this work non-commercially, and license their derivative works on different terms, provided the original work is properly cited, appropriate credit is given, any changes made indicated, and the use is non-commercial. See: http://creativecommons.org/licenses/by-nc/4.0/.

\section{ORCID iDs}

Jeremiah Seni http://orcid.org/0000-0003-2420-5329

Niranjan Konduri http://orcid.org/0000-0002-6828-1182

\section{REFERENCES}

1 WHO. Global antimicrobial resistance surveillance system (glass) report. early implementation 2016-2017, 2018. Available: https:// www.who.int/glass/resources/publications/early-implementationreport-2017-2018/en/

2 Levy SB. The 2000 Garrod lecture. factors impacting on the problem of antibiotic resistance. J Antimicrob Chemother Jan 2002;49:25-30.

3 WHO. World antibiotic awareness week, 13-17 November 2017. Geneva, Switzerland: World Heath Organization, 2017.

4 WHO. Joint external evaluation of IHR core capacities of the United Republic of Tanzania. Geneva: World Health Organization, 2017.

5 Gwimile JJ, Shekalaghe SA, Kapanda GN, et al. Antibiotic prescribing practice in management of cough and/or diarrhoea in Moshi Municipality, Northern Tanzania: cross-sectional descriptive study. Pan Afr Med J 2012;12:103.

6 Cox JA, Vlieghe E, Mendelson M, et al. Antibiotic stewardship in lowand middle-income countries: the same but different? Clin Microbiol Infect 2017;23:812-8.

7 WHO. Antimicrobial resistance: global report on surveillance. June 2014 ed. Geneva, Switzerland: World Heath Organization, 2014.

8 United Republic of Tanzania (URT). The National action plan on antimicrobial resistance (2017-2022). The Ministry of health community development gender elderly and children (MHCDGEC), Ministry of agriculture, livestock and fisheries (MalF). The United Republic of Tanzania 2017.

9 WHO. Global action plan on antimicrobial resistance. Geneva, Switzerland: The World Health Organization, 2015. http://apps. who. int/gb/ebwha/pdf_files/WHA68/A68_20-en.pdf?ua=1

10 Seni J, Najjuka CF, Kateete DP, et al. Antimicrobial resistance in hospitalized surgical patients: a silently emerging public health concern in Uganda. BMC Res Notes 2013;6:298.

11 Seni J, Mwakyoma AA, Mashuda F, et al. Deciphering risk factors for blood stream infections, bacteria species and antimicrobial resistance profiles among children under five years of age in northwestern Tanzania: a multicentre study in a cascade of referral health care system. BMC Pediatr 2019;19:32.
12 Sonda T, Kumburu H, van Zwetselaar M, et al. Meta-analysis of proportion estimates of extended-spectrum-beta-lactamaseproducing enterobacteriaceae in East Africa hospitals. Antimicrob Resist Infect Control 2016;5:18.

13 WHO. World Health Organization methodology for point prevalence survey on antibiotic use in hospitals, version 1.1. Geneva: World Health Organization, 2018.

14 Gharbi M, Doerholt K, Vergnano S, et al. Using a simple pointprevalence survey to define appropriate antibiotic prescribing in hospitalised children across the UK. BMJ Open 2016;6:e012675.

15 Talaat M, Saied T, Kandeel A, et al. A point prevalence survey of antibiotic use in 18 hospitals in Egypt. Antibiotics 2014;3:450-60.

16 Aldeyab MA, Kearney MP, McElnay JC, et al. A point prevalence survey of antibiotic prescriptions: benchmarking and patterns of use. $\mathrm{Br} J$ Clin Pharmacol 2011;71:293-6.

17 Versporten A, Zarb P, Caniaux I, et al. Antimicrobial consumption and resistance in adult hospital inpatients in 53 countries: results of an Internet-based global point prevalence survey. The Lancet Global Health 2018;6:e619-29.

18 Okoth C, Opanga S, Okalebo F, et al. Point prevalence survey of antibiotic use and resistance at a referral hospital in Kenya: findings and implications. Hosp Pract 2018;46:128-36.

19 WHO. World Health Organizaton collaborating centre for drug statistics methodology, ATC/DDD index 2017. Oslo: WHO Collaborating Centre for Drug Statistics Methodology, 2017. https:// www.whocc.no/atc ddd index/

20 WHO. The $2019 \mathrm{WHO}$ aware classification of antibiotics for evaluation and monitoring of use. World Health Organization, 2019. Available: https://apps.who.int/iris/handle/10665/327957

21 Anand Paramadhas BD, Tiroyakgosi C, Mpinda-Joseph P, et al. Point prevalence study of antimicrobial use among hospitals across Botswana; findings and implications. Expert Rev Anti Infect Ther 2019;17:535-46.

22 Oduyebo O, Olayinka A, Iregbu K. A point prevalence survey of antimicrobial prescribing in four Nigerian tertiary hospitals, 2017. Available: https://ir.unilag.edu.ng/handle/123456789/7155

23 Elhajji FD, Al-Taani GM, Anani L, et al. Comparative point prevalence survey of antimicrobial consumption between a hospital in Northern Ireland and a hospital in Jordan. BMC Health Serv Res 2018;18:849.

24 Porto APM, Goossens H, Versporten A, et al. Global point prevalence survey of antimicrobial consumption in Brazilian hospitals. $J$ Hosp Infect 2020;104:165-71.

25 Vandael E, Latour K, Goossens H, et al. Point prevalence survey of antimicrobial use and healthcare-associated infections in Belgian acute care hospitals: results of the Global-PPS and ECDC-PPS 2017. Antimicrob Resist Infect Control 2020;9:13.

26 Dlamini NN, Meyer JC, Kruger D, et al. Feasibility of using point prevalence surveys to assess antimicrobial utilisation in public hospitals in South Africa: a pilot study and implications. Hosp Pract 2019;47:88-95.

27 Sonda TB, Horumpende PG, Kumburu HH, et al. Ceftriaxone use in a tertiary care hospital in Kilimanjaro, Tanzania: a need for a hospital antibiotic stewardship programme. PLoS One 2019;14:e0220261.

28 Haldeman MS, Kishimbo P, Seddon M, et al. Evaluation of antimicrobial utilization and concordance with national guidelines at a tertiary hospital in the southern highlands zone of Tanzania. Am J Trop Med Hyg 2020;102:370-6.

29 URT. Standard treatment guidelines \& national essential medicines list. The Ministry of health, community development, gender, elderly and children. Fifth Edition. Tanzania: United Republic of Tanzania, 2017.

30 Sangeda RZ, Kibona J, Munishi C, et al. Assessment of implementation of antimicrobial resistance surveillance and antimicrobial stewardship programs in Tanzanian health facilities a year after launch of the National action plan. Frontiers in Public Health 2020;8:1-9.

31 Liu C, Liu C, Wang D, et al. Intrinsic and external determinants of antibiotic prescribing: a multi-level path analysis of primary care prescriptions in Hubei, China. Antimicrob Resist Infect Control 2019;8:132

32 Amaha ND, Weldemariam DG, Abdu N, et al. Prescribing practices using WHO prescribing indicators and factors associated with antibiotic prescribing in six community pharmacies in Asmara, Eritrea: a cross-sectional study. Antimicrob Resist Infect Control 2019;8:163.

33 WHO. World Health Oranization benchmarks for international health regulations (IHR) capacities. Geneva, Switzerland: WHO, 2019.

34 WHO. Antimicrobial stewardship programmes in health-care facilities in low- and middle-income countries. A practical toolkit. Geneva: World Health Organization, 2019. 